\title{
The mTOR inhibitor RAD001 augments radiation- induced growth inhibition in a hepatocellular carcinoma cell line by increasing autophagy
}

\author{
ANAÏS ALTMEYER ${ }^{1 *}$, ELODIE JOSSET $^{1 *}$, JEAN-MARC DENIS $^{2}$, JOHN GUEULETTE $^{2}$, \\ JAKOBUS SLABBERT $^{3}$, DIDIER MUTTER ${ }^{4}$, GEORGES NOËL ${ }^{1}$ and PIERRE BISCHOFF ${ }^{1}$ \\ ${ }^{1}$ EA 3430, University of Strasbourg, Centre Paul Strauss, Strasbourg, France; ${ }^{2}$ MIRO, \\ Catholic University of Louvain, Brussels, Belgium; ${ }^{3}$ iThemba LABS, Somerset West, \\ South Africa; ${ }^{4}$ Research Institute against Digestive Cancer (IRCAD), Strasbourg, France
}

Received March 8, 2012; Accepted May 9, 2012

DOI: 10.3892/ijo.2012.1583

\begin{abstract}
Treatment of hepatocellular carcinoma (HCC) is a major concern for physicians as its response to chemotherapy and radiotherapy remains generally poor, due, in part, to intrinsic resistance to either form of treatment. We previously reported that an irradiation with fast neutrons, which are high-linear energy transfer (LET) particles, massively induced autophagic cell death in the human HCC SK-Hep1 cell line. In the present study, we tested the capacity of the mammalian target of rapamycin (mTOR) inhibitor RAD001 to augment the cytotoxicity of low and high-LET radiation in these cells. As mTOR is a key component in a series of pathways involved in tumor growth and development, it represents a potential molecular target for cancer treatment. Results indicate that RAD001, at clinically relevant nanomolar concentrations, enhances the efficacy of both high- and low-LET radiation in SK-Hep1 cells, and that the induction of autophagy may account for this effect. However, fast neutrons were found to be more efficient at reducing tumor cell growth than low-LET radiation.
\end{abstract}

\section{Introduction}

Hepatocellular carcinoma (HCC) currently represents the sixth most common cancer in the world. Its incidence, very high in Asian countries, is steadily increasing in occidental countries. HCC is often refractory to radiation therapy (RT) and chemotherapy leaving surgery (followed by liver trans-

Correspondence to: Dr Pierre Bischoff, Université de Strasbourg, EA 3430, Centre Régional de Lutte Contre le Cancer Paul Strauss, 3 Rue de la Porte de l'Hôpital, F-67085 Strasbourg cedex, France E-mail: pbischoff@strasbourg.unicancer.fr

*Contributed equally

Key words: mTOR, RAD001, autophagy, high-LET radiation, hepatocellular carcinoma plantation) as the sole alternative to delay disease progression. As with other forms of cancer, the radioresistance of HCC cells stems, in part, from their intrinsic inability to undergo an apoptotic process in response to ionizing radiation (IR). The toxicity of IR on surrounding healthy hepatic tissue is another source of limitation for RT. Among other therapeutic strategies, two promising approaches to overcome the resistance of HCC cells to IR and conventional chemotherapy have been proposed: i) the utilization of high linear energy transfer (LET) radiation instead of low-LET radiation (1) and ii) the implementation of molecularly targeted drugs (2). High-LET based RT, or hadrontherapy, principally involves the use of accelerated carbon ions. The main advantage of charged high-LET particles is that they can deposit their energy within the tumor with extreme accuracy $(3,4)$. Although only a handful of hadrontherapy facilities currently exist worldwide, their number is slowly, but steadily, growing. The use of chemotherapeutic drugs that can be concomitantly associated with radiation offer another possibility of treatment for HCC. However, few such radiosensitizing drugs are currently available and their efficacy is limited. There is, therefore, an obvious need for novel radiosensitizing agents, more potent and molecularly targeted (5,6). In this context, RAD001 (everolimus), an inhibitor of the mammalian target of rapamycin (mTOR) could offer new perspectives in the treatments of HCC. Indeed, mTOR is a key downstream protein kinase in the PI3K/AKT pathway, which is commonly involved in tumorigenesis, including cancer cell growth and angiogenesis $(7,8)$. Aberrant hyperactivation of mTOR has been related to cancer progression (9) and is found in up to $60 \%$ of HCCs, which led to the recommendation of RAD001 for the treatment of this disease (10). Thus, it was interesting to evaluate the consequences of a combination between two modern, but non-standard treatments of HCC: high-LET radiation on the one hand, and a molecularly targeted anticancer drug on the other hand.

The present study was designed to assess the effect of RAD001 combined with low- and high-LET radiation upon the growth of SK-Hep1, a hepatic mesenchymal tumor cell line. For this purpose, we used fast neutrons as high-LET 
radiation. Although neutrons are now rarely utilized in therapy, they remain helpful as models for investigating the biological action of high-LET particles (11). We report here that the association of RAD001 with fast neutron irradiation resulted in greater antiproliferative and cytotoxic effects than the association of RAD001 with $\gamma$-irradiation. This indicates that high-LET radiation combined with RAD001 may provide an approach for HCC treatment that merits further evaluation.

\section{Materials and methods}

Reagents. RAD001 was a generous gift of Novartis (Basel, Switzerland). It was dissolved in DMSO at $40 \mu \mathrm{M}$ and stored at $-20^{\circ} \mathrm{C}$ and final dilutions were prepared extemporaneously in culture medium. Sulforhodamine B (SRB) and trichloroacetic acid (TCA) were purchased from Sigma-Aldrich (Saint-Quentin Fallavier, France).

Cell culture. The human HCC SK-Hep1 cell line was purchased from the American Type Culture Collection (ATCC, USA). Cultures were maintained at $37^{\circ} \mathrm{C}$ in a humid atmosphere of $5 \% \mathrm{CO}_{2}$. Cells were grown in Dulbecco's modified Eagle's medium (PAN Biotech $\mathrm{GmbH}$ ) supplemented with $10 \%$ fetal bovine serum (PAN Biotech $\mathrm{GmbH}$ ), $1 \mathrm{mM}$ sodium pyruvate, $1 \mathrm{mM}$ non-essential amino acids and $50 \mu \mathrm{g}$ penicillin-streptomycin (Life Technologies, Grand Island, USA). Disaggregation was carried out using $5 \mathrm{~min}$ incubation at $37^{\circ} \mathrm{C}$ with a solution of trypsin-EDTA (PAN Biotech $\mathrm{GmbH}$ ).

Irradiation procedure and treatment schedule. Asynchronous, exponentially growing cells were exposed at room temperature to low- or high-LET radiation. Cells were contained in 6-well plates filled with $4 \mathrm{ml}$ culture medium or in 96-flat bottomed microplates, filled with $0.2 \mathrm{ml}$ culture medium. Cells were treated with solvent control or RAD001 $1 \mathrm{~h}$ before irradiation. Irradiations with low-LET radiation were carried out with a ${ }^{137} \mathrm{Cs} \gamma$ irradiator (Biobeam GM8000, GSM Gmbh, Leipzig, Germany). Dose rate was $3.4 \mathrm{~Gy} / \mathrm{min}$ and doses ranged from 2 to 16 Gy. For high-LET radiation, we used $p(65)+$ Be neutrons produced by a cyclotron at the Cyclotron Resources Center (CRC) of Louvain-la-Neuve (Belgium) and at iThemba LABS (Faure, South Africa). Dose rate was usually $0.2 \mathrm{~Gy} / \mathrm{min}$ in both facilities, and doses ranged from 1 to $8 \mathrm{~Gy}$. Dose determination was performed using ionization chambers and thermo luminescent dosimeters (TLD). Each experiment was repeated at least three times.

Cell proliferation assay. The effects of the combined treatments on the growth of SK-Hep1 were investigated using the sulforhodamine B (SRB) colorimetric assay. Cells were seeded at a density of $5 \times 10^{3}$ cells/wells in $100 \mu \mathrm{l}$ in 96-flat bottomed well plates (Falcon 3072). Various dilutions of RAD001 (100 $\mu \mathrm{l})$ were added $24 \mathrm{~h}$ later to quintuplicate wells. Cells were then irradiated and incubated at $37^{\circ} \mathrm{C}$ for 2 , 6 or 9 days. They were then fixed with $10 \%$ TCA for $1 \mathrm{~h}$ at $4^{\circ} \mathrm{C}$, washed 5-fold with tap water, air dried and stained with $0.4 \% \mathrm{SRB}$ in $1 \%$ acetic acid for $30 \mathrm{~min}$. SRB-stained cells were then dissolved in $200 \mu 110 \mathrm{mM}$ Tris-base (pH 10.5) and the absorbance of each well was measured at $565 \mathrm{~nm}$ using an MRX microplate reader (Labtek, Issy-les-Moulineaux,
France). Results are expressed in optical density (OD), after subtractions of the blank (no cells).

Apoptotic and autophagic assays. Apoptotic cells were quantified according to a previous study (12). Briefly, cells $\left(5 \times 10^{5}\right)$ were fixed in cold $70 \%$ ethanol for at least $1 \mathrm{~h}$. Then, they were washed in phosphate buffered saline (PBS) pH 7.2 and resuspended in $100 \mu \mathrm{l}$ of PBS containing $25 \mu \mathrm{g}$ of RNase A, $2 \mathrm{mM}$ EDTA and $10 \mu \mathrm{g}$ of propidium iodide (PI). Following incubation in the dark for $30 \mathrm{~min}$ at $37^{\circ} \mathrm{C}$, the fluorescence of 10,000 cells was analyzed using a FACScan flow cytometer (Becton Dickinson, San Jose, CA) and Cell Quest software (Becton Dickinson). Cells with a sub-diploid DNA content were recorded as apoptotic.

For autophagy determination, we used the Cyto-ID ${ }^{\mathrm{TM}}$ Autophagy detection kit (Enzo Life Sciences, Plymouth Meeting, PA) according to the manufacturer's instructions. This test measures autophagic vacuoles and monitors autophagic flux in live cells using a novel fluorescent cationic amphiphilic dye that selectively labels autophagic vacuoles. Briefly, cells $\left(5 \times 10^{5}\right)$ were washed in PBS pH 7.2 and resuspended in $500 \mu \mathrm{l}$ of freshly diluted Cyto-ID ${ }^{\circledR}$ Green Detection Reagent $1 \mu \mathrm{l}$ to a final volume of $2 \mathrm{ml}$ with PBS. Following incubation in the dark for $30 \mathrm{~min}$ at $37^{\circ} \mathrm{C}$, the fluorescence of 10,000 cells was analyzed using a FACScan flow cytometer (Becton Dickinson). and Cell Quest software (Becton Dickinson).

Clonogenic survival assay. Twenty-four hours after exposure to fast neutrons or conventional, low-LET radiations, RAD001-treated and control cells were trypsinised and counted using a Countess ${ }^{\circledR}$ Cell Counter (Invitrogen). Unirradiated control cells were submitted to the same conditions. Irradiated and control cells were resuspended at an appropriate number in fresh medium and plated at two different dilutions into 6-well plates. Three wells were used by experimental point. Fifteen days later, colonies were stained with $0.5 \%$ crystal violet and colonies containing more than 50 cells were scored.

Analysis of $\gamma H 2 A X$ foci. SK-Hep1 cells were grown on microscopic glass slides placed in 6-well plates. Twenty-four hours post-irradiation, culture medium was removed and the slides were washed with PBS. Fixation and permeabilization were carried out using $4 \%$ paraformaldehyde and $0.5 \%$ Triton, respectively. Labeling was performed using a monoclonal mouse anti- $\gamma-\mathrm{H} 2 \mathrm{AX}$ antibody (clone JBW301, Upstate, Lake Placid, NY). Coverslips were mounted in 4',6-diamino-2-phenylindole (DAPI)-stained Vectashield (Abcys, Paris, France). The formation of $\gamma \mathrm{H} 2 \mathrm{AX}$ foci in nuclei was monitored by immunofluorescence microscope imaging. Foci were scored in at least 40 cells in each experimental condition.

Statistical analysis. Statistical analyses were performed using the MedCalc statistical software. Differences between the subgroups in terms of foci number and percentage of viable cell number in SRB assays with respect to the treatment and irradiation conditions were pair compared with a StudentNewman-Keuls. Differences were considered to be significant at $\mathrm{p}<0.05$. 


\section{Results}

Effect on cell growth and cell survival. We first evaluated the capacity of RAD001 to influence cell growth alone or combined with radiation. In a preliminary series of experiments, serial concentrations of RAD001, ranging from 1 to $40 \mathrm{nM}$, were added to SK-Hep1 in the absence of irradiation. They indicated that $20 \mathrm{nM}$ was sufficient to reduce cell growth while not affecting cell viability (not shown). Since this concentration is within the range of serum levels usually targeted to obtain efficacy with minimal toxicity in the clinic (13), it was selected for our subsequent experiments. We then assessed the consequences of a combination between RAD001 and an irradiation with low- or high-LET radiation. RAD001 was added to SK-Hep1 cells $1 \mathrm{~h}$ before exposure to either $\gamma$-rays or neutrons. SRB assays were performed at different days afterwards, generally at 6 days as this time interval proved to be optimal for obtaining a clear-cut difference between control and experimental groups. In irradiated, untreated cells, a marked reduction of cell growth was recorded at $8 \mathrm{~Gy}$ (photons) and 4 Gy (neutrons). In RAD001-treated cells, this decrease was slightly, but significantly more pronounced (Fig. 1). Indeed, the ratio: optical density (OD) of treated cells/OD of untreated cells x100 was 54\% in 8 Gy $\gamma$-irradiated cells and $49 \%$ in $4 \mathrm{~Gy}$ neutrons-irradiated cells. RAD001 also reduced cell growth in unirradiated cells but at a lesser extent (87\% of control, untreated cells). No clear-cut decrease of OD was observed according to the irradiation dose, indicating that $4 \mathrm{~Gy}$ (for neutrons) and 8 Gy (for photons) were sufficient for obtaining a significant reduction of the cell numbers.

In parallel, we performed clonogenic assays in order to assess the capacity of the various treatments to affect the replicative survival of SK-Hepl cells. As expected, neutrons were more cytotoxic than photons, since at 6 Gy the former were sufficient to almost entirely abrogate cell survival in the absence of RAD001 co-treatment (Fig. 2B). When associated to photons, RAD001 slightly decreased the survival fractions at the different irradiation doses (Fig. 2A). Of note, when combined to fast neutrons, no diminution of the survival fraction was achieved (Fig. 2B). With both types of radiation, the slope of the curve was identical for control and RAD001treated cells.

Effect on cell death induction. In further experiments, we attempted to identify the types of cell death caused by the association between RAD001 and radiation, and to quantify them. The percentage of cells undergoing apoptosis and autophagy were determined over a period of 6 days following irradiation. Results shown in Fig. 3 indicate that apoptosis can be detected 2 days following irradiation provided by either fast neutrons or photons but at low levels. Six days after exposure to the radiation, the percentage of apoptotic cells was higher, but did not exceed $25 \%$ in the control cells. Neutrons were no more efficient than photons at inducing apoptosis in SK-Hep1, confirming a previous study (14). In RAD001-treated cells, regardless of the radiation, apoptosis was reduced at different doses.

Furthermore, we tested the capacity of RAD001 to modify autophagy. We used a $488 \mathrm{~nm}$ excitable fluorescent reagent (Cyto-ID $^{\mathrm{TM}}$ Autophagy detection kit) that allows a conve-

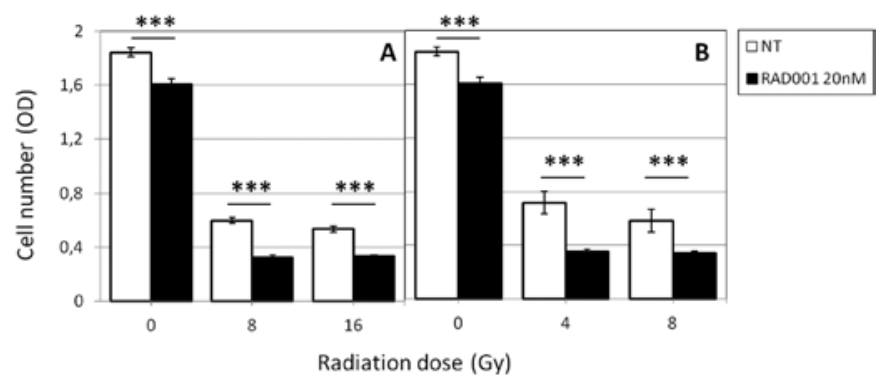

Figure 1. Effect of the combination of RAD001 (20 nM) and low and high-LET radiation on the growth of SK-Hep1 cells, as a function of the radiation dose. Sulforhodamine B (SRB) tests were performed 6 days after the irradiation, according to the protocol described in Materials and methods. (A) $\gamma$-irradiated cells, (B) neutron-irradiated cells. Cell numbers are expressed in optical density (OD). Bars represent the standard deviation (SD) of quintuplicate determination. ${ }^{* * *} \mathrm{p}<0.001$

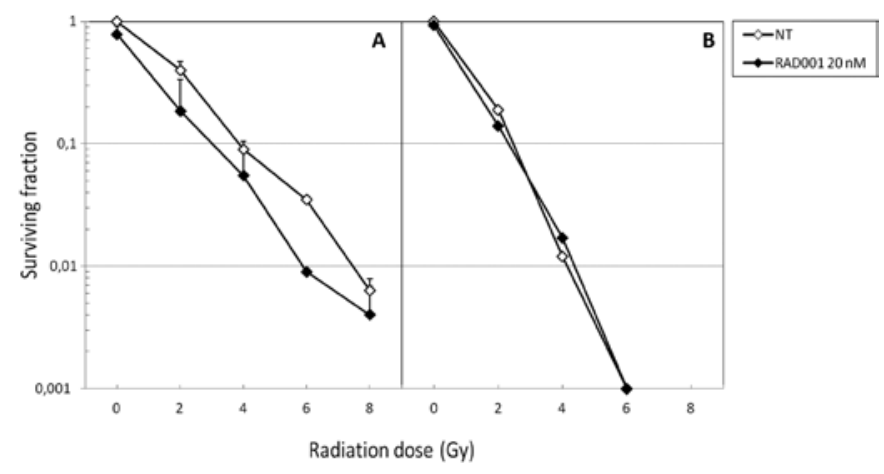

Figure 2. Clonogenic survival: surviving fraction of RAD001-treated, or untreated SK-Hep1 cells, irradiated by (A) $\gamma$-rays or (B) fast neutrons. Cells were treated with $20 \mathrm{nM}$ of RAD001 or DMSO (controls). Radiation doses ranged from 0 to $8 \mathrm{~Gy}$. Fifteen days after irradiation, colonies were stained with crystal violet and colonies containing more than 50 cells were scored.

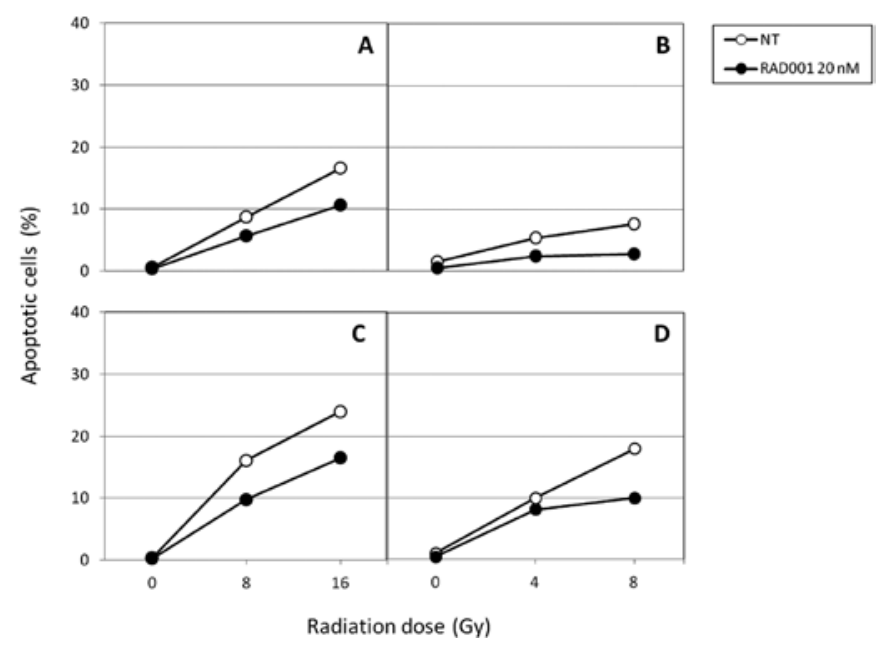

Figure 3. Induction of apoptosis in RAD001-treated or untreated SK-Hep1 cells, (A,B) 2 days and (C,D) 6 days after irradiation by $(A, C) \gamma$-rays and $(\mathrm{B}, \mathrm{D})$ neutrons. Cells were labeled with propidium iodide and the number of hypodiploid DNA-containing cells (expressed in \%) were determined by flow cytometry.

nient quantification of autophagic cells by flow cytometry. In preliminary experiments, we validated the ability of this assay to record autophagy, by comparing results with other methods, 
such as GFP-LC3 expression in autophagosomes and electron microscopy. As shown in Fig. 4A, photon-induced autophagy levels were high 2 days following irradiation in untreated cells, since $60 \%$ of cells were positive at 8 Gy and 16 Gy. Six days following irradiation, these values decreased appreciably, since at the same doses, only 25 and $18 \%$ cells were autophagic at 8 and 16 Gy respectively. In neutron-irradiated cells (Fig. 4B), the same patterns were observed according to the dose, but at doses 2-fold lower. In RAD001-treated cells, a marked increase of autophagy in both photon- and neutron-irradiated ones was obtained 6 days post-irradiation, as compared with untreated ones, suggesting that RAD001 induced a sustained level of autophagy in irradiated cells. Markedly, when used alone at $20 \mathrm{nM}$, RAD001 was unable to induce autophagy at either 2 or 6 days of culture. This lack of autophagy induction in RAD001-treated, unirradiated cells was confirmed using green fluorescence protein (GFP)-LC3 staining (not shown). It may reflect cell type differences, since the same RAD001 concentration could induce autophagy in U87 glioblastoma cells (not shown), which is consistent with other recently published data (15).

$H 2 A X$ foci determination. Radiation lethality results mainly from the generation of double-strand breaks (DSBs) in DNA. If irradiated cells fail to repair such lesions, they undergo a death program. Apoptosis is generally initiated, but autophagy can also be induced by DNA damage (16). We therefore compared the capacity of RAD001 to interact with the formation and the repair of DSBs, using the detection of $\gamma \mathrm{H} 2 \mathrm{AX}$ foci as an end point. This method gives valuable information on the persistence of DSBs, hence their lack of repair. As expected, the number of persistent foci at $24 \mathrm{~h}$ post-irradiation augmented with the irradiation dose, and was found to be, at the same dose, approximately 2-fold higher in neutrons irradiated cells than in low-LET irradiated ones (Fig. 5A). In RAD001-treated cells, the numbers of foci were lower than in untreated cells, albeit not significantly (Fig. 5B).

\section{Discussion}

We evaluated the cytotoxic consequences of combinations between the mTOR inhibitor RAD001 and high and low-LET radiation in SK-Hep1 HCC cells. We report here that this cotreatment caused a marked decrease of their growth. We also found that in irradiated SK-Hep1 cells, death occurred by autophagy instead of apoptosis, confirming previous results obtained in vitro (14) and in vivo, after orthotopic transplantation of these cells into nude mice (17). As previously noted, high-LET radiation offers several significant advantages over low-LET radiation. First of all, they provoke more deleterious damage in DNA than sparsely ionizing radiation resulting from the fact that high-LET particles produce dense ionization along their trajectories, causing clustered and complex damage to DNA, known as 'locally multiple damaged sites' (LMDS) and also spoiling intracellular structures $(4,18)$. Beyond a given threshold of LMDS, the capacity of DNA repair machinery is overwhelmed, leading to cell death. As a consequence, high-LET radiation can inactivate or kill malignant cells more effectively than low-LET radiation, by inducing premature senescence, apoptosis, necrosis and autophagy (4).

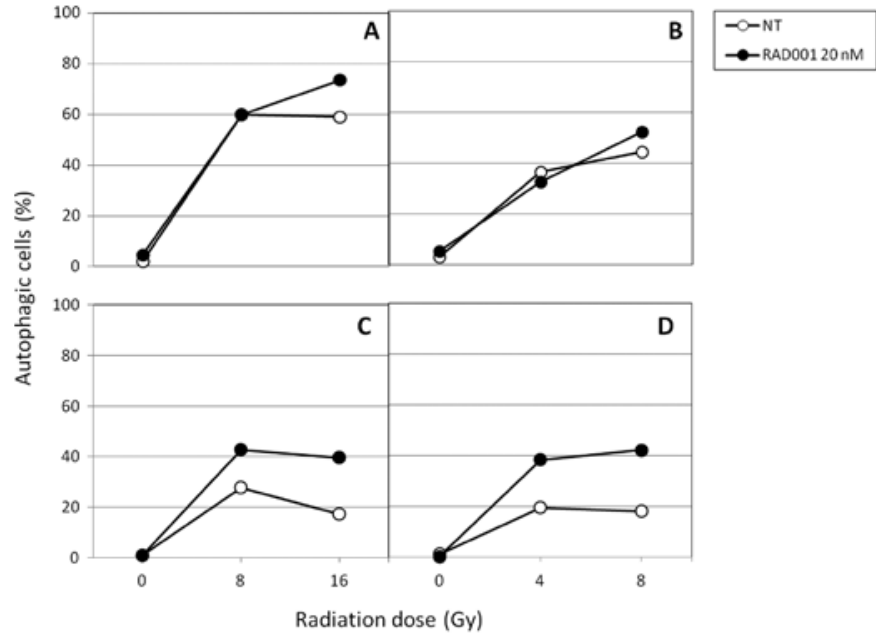

Figure 4. Induction of autophagy in RAD001-treated or untreated SK-Hep1 cells, (A,B) 2 days and (C,D) 6 days after irradiation by $(A, C) \gamma$-rays and $(B, D)$ neutrons. Percentages of autophagic cells were determined by flow cytometry, using the Cyto-ID ${ }^{\mathrm{TM}}$ autophagy detection kit according to the procedure described in Materials and methods.

Therefore, it was interesting to compare the effects of these two types of radiation when combined with RAD001. As expected, at the same physical dose, neutrons were found to be more efficient than photons at inducing autophagy and decreasing proliferation.

In fact, we previously reported that autophagy principally accounted for the loss of cell survival in $\operatorname{HCC}(14,17)$ and glioblastoma (GBM) (19) neutron-irradiated cell lines alone, or associated with oxaliplatin. Currently, whether autophagy constitutes a mode of protection or, conversely, contributes to cell death is not firmly recognized, and the consequences of its induction by IR in radiation therapy are still actively debated (20). However, it must be pointed out that autophagy implies a series of progressive cellular processes, and that cell death is induced by an excessive and extreme form of autophagy (21). Thus, possibly more than with apoptosis, which is an all or nothing phenomenon, the pharmacological modulation of autophagy might lead to a differential effect of radiation between normal and malignant cells. Another aspect of autophagy induction in tumor cells can be envisioned. Indeed, it has recently been reported that autophagosomes could play a key role in the presentation of tumor antigens, and that autophagosomes were efficient carriers for priming CD8 lymphocytes (22). On the other hand, it has been pointed out that local radiation therapy could inhibit tumor growth through the generation of tumor-specific cytotoxic T lymphocytes (CTL)(23). Thus, we could assume that, by inducing autophagy instead of apoptosis, radiation could exert its action by activating the immune defenses of the organism. Clearly, in cultured cells, such a mechanism cannot account for the radiosensitizing effect. However, the importance of mTOR inhibition in enhancing radiation-induced autophagy has already been emphasized (24). The efficacy of RAD001 at augmenting the sensitivity of malignant cells to radiation has been reported for several types of cancer, including lymphoma (25) and pituitary adenoma (26). The inhibition of mTOR has also been shown to enhance the radiosensitivity of some, but not all, tumor cell lines. For instance, an increase of radiosensitivity by mTOR 

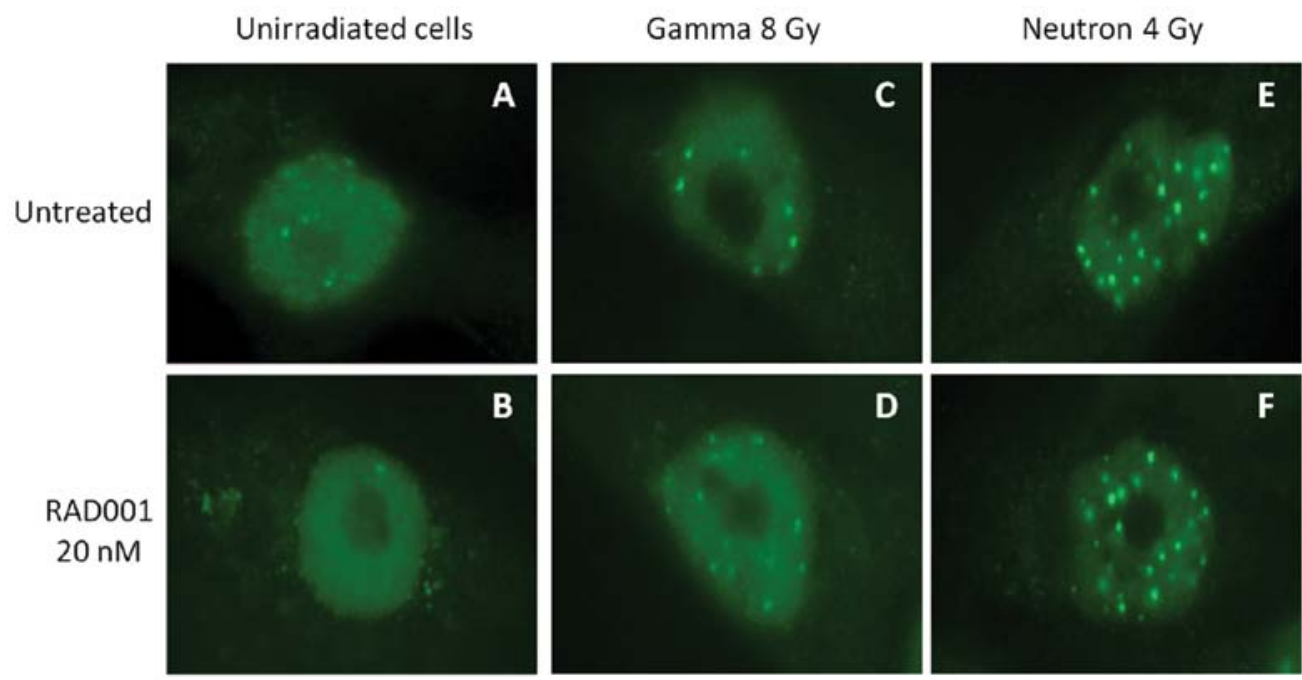

B

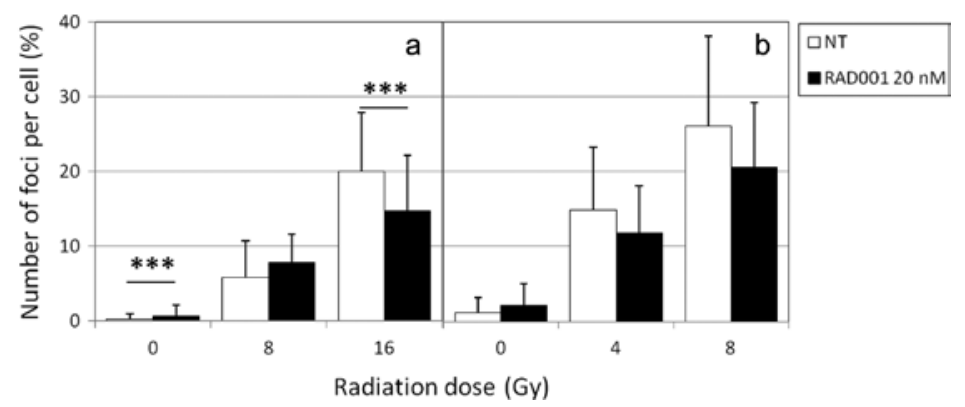

Figure 5. (A) Foci corresponding to the histone $\gamma \mathrm{H} 2 \mathrm{AX}$ activation in SK-Hep1 cells $24 \mathrm{~h}$ after exposure to 4 Gy $\gamma$-rays, 4 Gy fast neutrons, or left unirradiated. Upper row of pictures, untreated cells; lower row of pictures, RAD-001 treated cells. (B) Number of foci of $\gamma \mathrm{H} 2 \mathrm{AX} / \mathrm{nucleus}$ as a function of the dose and the radiation type. (a) $\gamma$-rays (b) neutrons. Foci were scored $24 \mathrm{~h}$ after irradiation. ${ }^{* * *} \mathrm{p}<0.001$.

inhibition has been observed in MCF-7 and MDA-MB-231, two human breast cancer cell lines (27). In HepG2-R, a radioresistant subline derived from the HCC HepG 2 cell line, the enhancement of autophagy by rapamycin could also result in radiosensitization and it was suggested that insufficient IR-induced autophagy may account for the radioresistance of these cells (28). However, this assumption cannot be extrapolated to all cancer cell lines since, in some cases, the inhibition of mTOR failed to significantly affect the radiation response. For example, a lack of radiosensitizing effect of rapamycin was noted in the GBM cell lines U87 and SKMG-3, as addition of this drug to the cells $24 \mathrm{~h}$ prior to irradiation had no effect on their clonogenic survival (29). In the mouse GL261 glioma cell line also, pretreatment with either rapamycin $(100 \mathrm{nM})$ or RAD001 (5 $\mathrm{nM})$ for $1 \mathrm{~h}$ before irradiation did not significantly enhance cell death over that caused by IR alone, as demonstrated by clonogenic assays in vitro (30). Recently, evidence was provided that inhibition of mTOR may also decrease rather than increase the radiosensitivity of tumor cells in vitro. This was shown in the case of the HeLa cervical adenocarcinoma cell line, after pretreatment for 3 or $24 \mathrm{~h}$ with rapamycin $(50 \mathrm{nM})$ followed by IR exposure and evaluation in a clonogenic cell survival assay. Notably, the increased radioresistance was only observed when rapamycin was added to the cells before irradiation and not if it was added at the same time (31).

Whether damage to DNA is involved in the radiosensitizing effect of RAD001 should also be considered. Indeed, rapamycin and $\mathrm{RAD} 001$ have been demonstrated to enhance the sensitivity of the HCC cell line Hep3B to cisplatin (32), which is known to exert cytotoxicity by inducing lesions in DNA. Moreover, rapamycin has recently been reported to suppress DSB repair in MCF-7 breast cancer cells (33). However, our results clearly indicate that the number of persistent DSBs after irradiation is not substantially altered in RAD001-treated SK-Hep1 cells, indicating that the drug is unlikely to affect the efficiency of DNA repair in these cells. The balance between the events leading to a defined cell death program, the PI3K/ Akt/mTOR pathways and the DNA damage repair pathways may also account for differences between cell lines. As these two pathways can vary according to the mutational status of the tumor cell lines, further studies using different tumor cell types are required to clarify the relationship between mTOR inhibition and the initiation of a specified form of cellular demise in irradiated cells.

In summary, the present data provide evidence that the association of RAD001 with high-LET radiation is effective in reducing the growth of HCC cells. They also suggest that a reinforcement of autophagy contributes to this effect. Thus, it should be determined whether RAD001, in combination with high-LET radiation, induced a sustained level of autophagy in other HCC cell lines as well as in tumor cells from other origins. Since RAD001 is well tolerated in patients with advanced HCC (34), its association with high-LET radiation merits further investigation in order to reach potential clinical evaluation. 


\section{Acknowledgements}

We thank Dr Francis J. Dumont for reviewing the manuscript.

\section{References}

1. Orecchia R, Krengli M, Jereczek-Fossa BA, Franzetti S and Gerard JP: Clinical and research validity of hadrontherapy with ion beams. Crit Rev Oncol Hematol 51: 81-90, 2004.

2. Finn RS: Development of molecularly targeted therapies in hepatocellular carcinoma: where do we go now? Clin Cancer Res 15: 390-397, 2010

3. Pommier P, Hu Y, Baron MH, Chapet O and Balosso J: Particle therapy: carbon ions. Bull Cancer 97: 819-829, 2010.

4. Hamada N, Imaoka T, Masunaga S, et al: Recent advances in the biology of heavy-ion cancer therapy. J Radiat Res 51: 365-383, 2010.

5. Dumont F, Altmeyer A and Bischoff P: Radiosensitising agents for the radiotherapy of cancer: novel molecularly targeted approaches. Expert Opin Ther Pat 19: 775-799, 2009.

6. Begg AC, Stewart FA and Vens C: Strategies to improve radiotherapy with targeted drugs. Nat Rev Cancer 11: 239-253, 2011.

7. Abraham RT and Gibbons JJ: The mammalian target of rapamycin signaling pathway: twists and turns in the road of cancer therapy. Clin Cancer Res 13: 3109-3114, 2007.

8. Chiong E, Lee IL, Dadbin A, et al: Effects of mTOR inhibitor everolimus (RAD001) on bladder cancer cells. Clin Cancer Res 17: 2863-2873, 2011

9. Dancey J: mTOR signaling and drug development in cancer. Nat Rev Clin Oncol 7: 209-219, 2010.

10. Kudo M: mTOR inhibitor for the treatment of hepatocellular carcinoma. Dig Dis 29: 310-315, 2011.

11. Gueulette J, Slabbert JP, Bischoff P, Denis JM, Wambersie A and Jones D: Fast neutrons: Inexpensive and reliable tool to investigate high-LET particle radiobiology. Rad Meas 45: 1414-1416, 2010.

12. Riccardi C and Nicoletti I: Analysis of apoptosis by propidium iodide staining and flow cytometry. Nature Protocols 1: $1458-1461,2006$

13. Xu B, Wu Y, Shen L, et al: Two-dose-level confirmatory study of the pharmacokinetics and tolerability of everolimus in Chinese patients with advanced solid tumors. J Hematol Oncol 13: 4-3, 2001.

14. Altmeyer A, Jung AC, Ignat M, et al: Pharmacological enhancement of autophagy induced in a hepatocellular carcinoma cel line by high-LET radiation. Anticancer Res 30: 303-310, 2010.

15. Nyfeler B, Bergman P, Triantafellow E, et al: Relieving autophagy and 4EBP1 from rapamycin resistance. Mol Cell Biol 31: 2867-2876, 2011

16. Rodriguez-Rocha H, Garcia-Garcia A, Panayiotidis MI and Franco R: DNA damage and autophagy. Mutat Res 3: 158-166, 2011.

17. Altmeyer A, Ignat M, Denis JM, Messaddeg N, Gueulette J, Mutter D and Bischoff P: Cell death after high-LET irradiation in orthotopic human hepatocellular carcinoma in vivo. In vivo 25: $1-9,2011$
18. Hada $\mathrm{M}$ and Georgakilas AG: Formation of clustered DNA damage after High-LET irradiation: a review. J Radiat Res 49: 203-210, 2008.

19. Benzina S, Altmeyer A, Malek F, et al: High-LET radiation combined with oxaliplatin induces autophagy in U-87 glioblastoma cells. Cancer Lett 264: 63-70, 2008.

20. Zois CE and Koukourakis MI: Radiation-induced autophagy in normal and cancer cells: towards novel cytoprotection and radiosensitization policies? Autophagy 5: 442-450, 2009.

21. Todde V, Veenhuis M and van der Klei IJ: Autophagy: Principles and significance in health and disease. Biochim Biophys Acta 1792: 3-13, 2009.

22. Li Y, Wang LX, Pang P, et al: Tumor-derived autophagosomes vaccine: mechanism of cross-presentation and therapeutic efficacy. Clin Cancer Res 17: 7047-7057, 2001.

23. Takeshima T, Chamoto K, Wakita D, et al: Local radiation therapy inhibits tumor growth through the generation of tumorspecific CTL: its potentiation by combination with Th1 cell therapy. Cancer Res 70: 2697-2706, 2010.

24. Jabouin JJ, Shinohara ET, Moretti L, Yang ES, Kaminski JM and $\mathrm{Lu}$ B: The role of mTOR inhibition in augmenting radiation induced autophagy. Technol Cancer Res Treat 6: 443-447, 2007.

25. Saunders P, Cisterne A, Weiss J, Bradstock KF and Bendall LJ: The mammalian target of rapamycin inhibitor RAD001 (everolimus) synergizes with chemotherapeutic agents, ionizing radiation and proteasome inhibitors in pre-B acute lymphocytic leukemia. Haematologica 96: 69-77, 2011.

26. Sukumari-Ramesh S, Singh N, Dhandapani KM and Vender JR mTOR inhibition reduces cellular proliferation and sensitizes pituitary adenoma cells to ionizing radiation. Surg Neurol Int 2: 22, 2011.

27. Albert JM, Kim KW, Cao C and Lu B: Targeting the Akt/ mammalian target of rapamycin pathway for radiosensitization of breast cancer. Mol Cancer Ther 5: 1183-1189, 2006.

28. Kuwahara Y, Oikawa T, Ochiai Y, et al: Enhancement of autophagy is a potential modality for tumors refractory to radiotherapy. Cell Deat Dis 2: 1-11, 2011.

29. Eshleman JS, Carlson BL, Madek AC, Kastner AD, Shide L and Sarkaria JN: Inhibition of the mammalian target of rapamycin sensitizes U87 xenografts to fractionated radiation therapy. Cancer Res 15: 7291-7297, 2002.

30. Shinohara ET, Cao C, Niermann K, Mu Y, Zeng F, Hallahan DE and $\mathrm{Lu} \mathrm{B}$ : Enhanced radiation damage of tumor vasculature by mTOR inhibitors. Oncogene 24: 5414-5422, 2005.

31. Bandhakavi S, Kim YM, Ro SH, et al: Quantitative nuclear proteomics identifies mTOR regulation of DNA damage response. Mol Cell Proteomics 9: 403-414, 2010.

32. Tam KH, Yang ZF, Lam CT, Pang RW and Poon RT: Inhibition of mTOR enhances chemosensitivity in hepatocellular carcinoma. Cancer Lett 273: 201-209, 2009.

33. Chen H, Vanderwaal RP, Feng Z, et al: The mTOR inhibitor rapamycin suppresses DNA double-strand break repair. Radiat Res 175: 214-224, 2011.

34. Zhu AX, Abrams TA, Miksad R, et al: Phase 1/2 study of everolimus in advanced hepatocellular carcinoma. Cancer 117 5094-5102, 2011 